\title{
Citation bias in the literature on dietary trans fatty acids and serum cholesterol
}

Citation for published version (APA):

Urlings, M. J. E., Duyx, B., Swaen, G. M. H., Bouter, L. M., \& Zeegers, M. P. A. (2019). Citation bias in the literature on dietary trans fatty acids and serum cholesterol. Journal of Clinical Epidemiology, 106, 88-97. https://doi.org/10.1016/j.jclinepi.2018.10.008

Document status and date:

Published: 01/02/2019

DOI:

10.1016/j.jclinepi.2018.10.008

Document Version:

Publisher's PDF, also known as Version of record

Document license:

Taverne

Please check the document version of this publication:

- A submitted manuscript is the version of the article upon submission and before peer-review. There can be important differences between the submitted version and the official published version of record.

People interested in the research are advised to contact the author for the final version of the publication, or visit the DOI to the publisher's website.

- The final author version and the galley proof are versions of the publication after peer review.

- The final published version features the final layout of the paper including the volume, issue and page numbers.

Link to publication

\footnotetext{
General rights rights.

- You may freely distribute the URL identifying the publication in the public portal. please follow below link for the End User Agreement:

www.umlib.nl/taverne-license

Take down policy

If you believe that this document breaches copyright please contact us at:

repository@maastrichtuniversity.nl

providing details and we will investigate your claim.
}

Copyright and moral rights for the publications made accessible in the public portal are retained by the authors and/or other copyright owners and it is a condition of accessing publications that users recognise and abide by the legal requirements associated with these

- Users may download and print one copy of any publication from the public portal for the purpose of private study or research.

- You may not further distribute the material or use it for any profit-making activity or commercial gain

If the publication is distributed under the terms of Article $25 \mathrm{fa}$ of the Dutch Copyright Act, indicated by the "Taverne" license above, 


\title{
Citation bias in the literature on dietary trans fatty acids and serum cholesterol
}

\author{
Miriam J.E. Urlings ${ }^{\mathrm{a}, \mathrm{b}, *}$, Bram Duyx ${ }^{\mathrm{a}, \mathrm{b}}$, Gerard M.H. Swaen ${ }^{\mathrm{a}, \mathrm{b}}$, Lex M. Bouter ${ }^{\mathrm{c}, \mathrm{d}}$, Maurice \\ P.A. Zeegers ${ }^{\mathrm{a}, \mathrm{b}}$ \\ ${ }^{\mathrm{a}}$ CAPHRI School for Public Health and Primary Care, Maastricht University, Maastricht, The Netherlands \\ ${ }^{\mathrm{b}}$ NUTRIM School of Nutrition and Translational Research in Metabolism, Maastricht University, Maastricht, The Netherlands \\ ${ }^{\mathrm{c}}$ Department of Epidemiology and Biostatistics, Amsterdam University Medical Centers, The Netherlands \\ ${ }^{\mathrm{d} D e p a r t m e n t}$ of Philosophy, Faculty of Humanities, Vrije Universiteit, Amsterdam, The Netherlands \\ Accepted 9 October 2018; Published online 19 October 2018
}

\begin{abstract}
Objective: Balanced citations are a necessary condition for a sound development of scientific knowledge, whereas selective citations may bias scientific consensus. In this study, we assess which determinants influenced the likelihood of being cited in the literature on trans fatty acids and cholesterol.

Study Design and Setting: We conducted a citation network analysis of the literature concerning trans fats and low density cholesterol and high density cholesterol. Each publication was scored on various potential determinants of citation, such as study outcome, study design, sample size, journal impact factor, and funding source. We applied random effect logistic regression to identify determinants of citation.

Results: A network of 108 publications was identified, containing 5,041 potential citation paths and 669 utilized citation paths. Reporting statistically significant results was found to be a strong predictor of citation, together with sample size, journal impact factor, and the authority of the authors.

Conclusion: Within the literature on trans fat intake and cholesterol, selective citations are based on several grounds. Especially the effect of reporting significant results on citation requires special attention because disproportionate attention is paid to publications suggesting a harmful effect of trans fat on cholesterol. (C) 2018 Elsevier Inc. All rights reserved.
\end{abstract}

\section{Introduction}

Citations are a key element in the development of knowledge and are highly valued within the scientific community. Citations have been suggested as a measure of (intellectual) influence [1,2], persuasiveness [3,4], and socially defined quality $[5,6]$. With the growing amount of literature in biomedical research, it is impossible to cite every relevant source and therefore only a selection of the relevant literature is cited in each publication. It might be questioned whether citation practices are solely driven by

Conflict of interest statement: The authors have no conflict of interest to declare.

* Corresponding author: Department of Complex Genetics, Maastricht University, P.O. Box 616, 6200 MD Maastricht, The Netherlands. Tel.: +31433881274.

E-mail address: mje.urlings@maastrichtuniversity.nl (M.J.E. Urlings). research quality, or whether factors such as the study results also influence selection of citations. Previous research, on clinical trials from a variety of fields, showed that most trials only cite a very small percentage of available previous trials and thereby neglect evidence [7,8]. Song (2010) defined the phenomenon when the selection of citations is related to the study result, as citation bias [9]. Citation bias is considered to be a questionable research practice (QRP). QRPs are a variety of scientific misbehaviors that negatively influence the development of knowledge [10]. A recent survey among scientists showed that selective citation is one of the most frequent QRPs [11].

The occurrence of citation bias has been studied in a number of research areas. Recently, a systematic review and meta-analysis summarized the literature on citation bias [12]. Forty-six publications on citation bias were identified from different research areas and using different methods. Overall, our systematic review concluded that 


\section{What is new?}

\section{Key findings}

- Citation bias is present in the literature on dietary trans fatty acids and serum cholesterol. Studies reporting a significant association between trans fat intake and LDL-c have a three times higher likelihood of being cited, compared to studies with nonsignificant results. Sample size, journal impact factor, and the authority of the author are also important determinants of selective citation.

\section{What this adds to what is known?}

- A multitude of research has proven that negative studies are cited less than positive studies. This research has also shown that multiple factors influence the likelihood of being cited.

- Citation network analysis has been developed to gain insight into the determinants of selective citation.

\section{What is the implication and what should change now?}

- Awareness should be raised that selective citation might drive the viewpoint of the reader in a certain direction and influence agenda setting, which might not be in line with the available evidence.

- Especially when science is being used in decisionmaking processes, more attention should be paid to reporting all of the available literature instead of relying on often cited publications.

citation bias is present, with the most convincing evidence from the field of biomedical sciences [12]. A meta-analysis in our review showed that positive studies are approximately two times more likely to be cited than negative studies. However, heterogeneity was very high because of the different methodologies and topics under study. Some publications studied citation bias in a specific research area [13-16], whereas others studied citation bias within one or more journals or databases [17-19] or assessed citation bias in systematic reviews [20-22]. In addition, the way in which citation bias was measured varied across publications. For example, Greenberg (2009) compared the percentage of positive citations to the percentage of negative citations in a certain field [23]. Ioannidis (2011) followed a different approach, by taking a cohort of publications that received more than 400 citations and comparing their reported effect sizes with the effect sizes in meta-analyses in the same field [24]. Both approaches assess the relationship between study outcome and citation but each from a different perspective.
Citation bias focuses on study outcome as the determinant of selective citation. However, selective citation can be driven by other factors as well. Determinants that have shown a positive association with citation rate in multiple studies are sample size, study design, journal impact factor, and the number of references $[19,25,26]$. Furthermore, privately funded studies are often believed to be less credible compared with publicly funded research [27]. Research has indicated that for-profit studies receive higher numbers of citations and thereby potentially skew knowledge development [28]. Other factors that have been incidentally linked to citation count, but with uncertain results, are gender of the author, number and type of affiliations involved in a publication, the authors' reputation, and whether the title of the publication includes its conclusion or not $[25,26]$. Finally, the language of a publication might influence the likelihood of citation, for example, by formulating firm conclusions or not. This can be measured as "hedging." Hedging refers to the use of vague language and therefore attenuates the strength of a claim [29]. Via specialized software [29], each publication can be given a hedging score between 0 and 5, with a higher value corresponding to the use of more uncertain language. To which extent these potential determinants of selective citation actually influence knowledge development is unclear and will most likely be subtle.

In the present study, we apply a new methodology to study the occurrence and determinants of selective citation. Inspired by previous literature, we have combined several approaches to assess the impact of different determinants on the likelihood of being cited. In this citation network analysis, we are not interested in the content or the correctness of citations but solely in the occurrence of selective citations in the light of skewed knowledge development. We aim to add to previous literature by providing a broader overview of selective citation by including all previously mentioned determinants into one study.

As an example topic, we will apply our methodology to the literature on the effect of industrially produced trans fatty acid (IP-TFA) intake on LDL cholesterol (LDL-c) and HDL cholesterol (HDL-c). IP-TFA is known to be associated with increased serum LDL-c and decrease serum HDL-c [30-33]. This scientific consensus has been strong enough for policy makers and industry to take action and limit the amount of IP-TFA in food $[34,35]$. The reason for choosing this research area as an example is that no obvious signs of citation bias are present in this field. Therefore, we can study the occurrence of citation bias and the determinants of selective citation in an objective way, without expectations with regard to the outcome. In addition, the fact that the discussion has been settled makes it interesting to see how selective citation might have influenced the development of consensus in this field.

\section{Methods}

Before performing the study, the citation network analysis method was described in a study protocol (see 
Supplementary Material). The main activities in the citation network analysis are also presented in this paragraph.

\subsection{Search strategy and article selection}

A systematic search strategy was performed in Web of Science Core Collections. The following search terms were used to identify relevant studies on IP-TFA, LDL-c, and HDL-c: ("Trans fat*" OR "Hydrogenated oil*" OR "Elaidic acid*") AND ("Low density lipoprotein" OR "High density lipoprotein" OR "Cardiovascular" OR "Coronary heart disease" OR "LDL" OR "HDL" OR "CVD"). The search strategy was based on broad search terms, to make sure no relevant publications would be missed. Reference lists of the identified articles were not checked for missing publications because this would interfere with the study aim. This would have caused an overrepresentation of articles cited within the network, whereas articles neglected by the network would less likely be found.

Publications were included if one of their study aims concerned the effect of IP-TFA intake on LDL-c and/or HDL-c and if the study design was an observational or an intervention study, a systematic review, or another type of synthesis article. Only studies on human subjects were included; no restrictions with regard to language or publication year were used.

The search, conducted by MJEU in January 2016, identified 1027 publications (Fig. 1). After the title selection, performed in duplo by MJEU and BD, 332 publications remained. A second selection round, based on abstract, performed by MJEU and GMHS, limited the number of publications to 118. Ultimately, the network contained 108 publications because the full text of 10 publications could not be identified, despite multiple attempts to find them via Google Scholar, the corresponding author and ResearchGate.

\subsection{Data extraction}

All publications in the network were scored on a number of potential determinants of citation, listed in Table 1. Data extraction was performed in duplo by MJEU and GMHS.

Study outcome was operationalized in two ways. First, we scored whether the publication reported a statistically significant effect of trans fat intake on LDL-c, HDL-c, or the ratio of total cholesterol:HDL-cholesterol (TC:HDL-c ratio). The latter was not described in our study protocol but added as an outcome measure because this appeared to be an important and often used biomarker of cardiovascular disease during the 1990's. Second, study outcome was measured by scoring whether or not the findings of the publication were in line with the hypotheses that IP-TFA intake increases LDL-c, decreases HDL-c, and increases the TC:HDL-c ratio. When the findings were in line with the previously mentioned hypotheses, scoring was based on

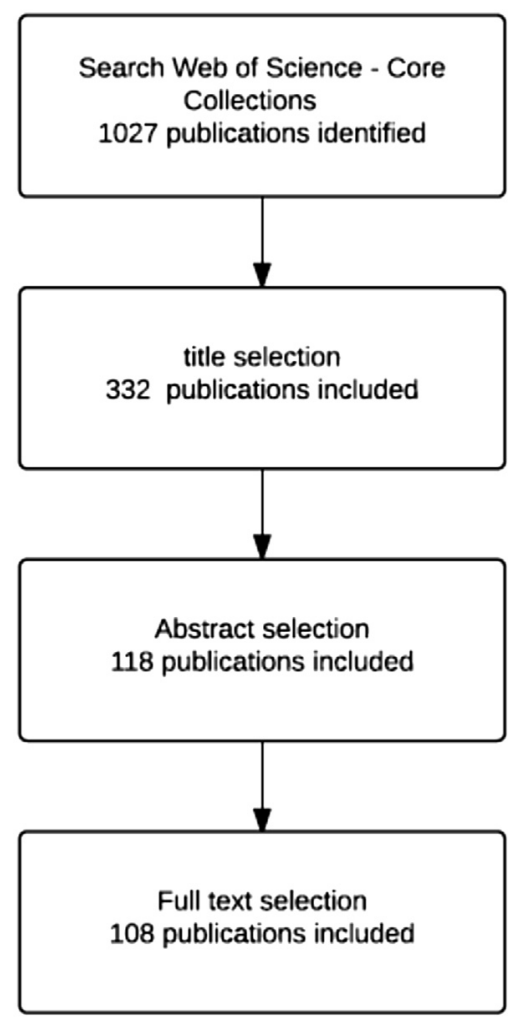

Fig. 1. Flow diagram of the article selection procedure.

the reported point estimate, without taking into account the confidence interval. For publications that did not report statistical results, such as narrative reviews, scoring was based on the author's conclusion. The study designs presented in this network were observational studies (cohort and cross-sectional studies), intervention studies, systematic reviews, narrative reviews, and editorials. Sample size was measured as the total number of participants included in a study. For systematic reviews, the sample size was measured as the sum of participants of all included studies. Narrative reviews or other documents where the number of participants was not clearly described were coded as having zero participants, to avoid missing values in the analysis. The determinant "authority of the corresponding author" was measured by the publication's level and varies over time. All coauthors of all publications were assigned an "authority score," which was the number of citations received within this IP-TFA network, at each year the network was active. The authority of the author was measured as the total number of citations received at the moment before a new potential citation, to avoid interference between the authority and the citation score.

Other variables that were measured are hedge factor, number of affiliations, journal impact factor at the moment of publication, funding source, number of references, whether or not the title of the publication described the conclusion of the publication, as well as the gender and the affiliation of the corresponding author. 
Table 1. Distribution of article characteristics and potential determinants of citation in the literature on trans fats and cholesterol of 108 articles and 5041 citation relations $^{a}$

\begin{tabular}{|c|c|c|c|}
\hline Article characteristic & N Publications & $N$ citation paths utilized (\%) & $N$ citation paths not utilized (\%) \\
\hline \multicolumn{4}{|l|}{ Study outcome } \\
\hline \multicolumn{4}{|l|}{ Statistical significance LDL-c } \\
\hline Significant increase LDL-c & 26 & $431(29)$ & $1,036(71)$ \\
\hline No significant increase LDL-c & 16 & $118(13)$ & $811(87)$ \\
\hline \multicolumn{4}{|l|}{ Statistical significance HDL-c } \\
\hline Significant decrease HDL-c & 21 & $308(27)$ & $852(73)$ \\
\hline No significant decrease HDL-C & 22 & $243(19)$ & $1,053(81)$ \\
\hline \multicolumn{4}{|l|}{ Statistical significance ratio TC:HDL-C } \\
\hline Significant increase TC:HDL-C & 12 & $123(25)$ & $378(75)$ \\
\hline No significant increase TC:HDL-C & 6 & $61(18)$ & $279(82)$ \\
\hline \multicolumn{4}{|l|}{ Hypothesis LDL-C } \\
\hline In line hypothesis-increase LDL-c & 86 & $513(14)$ & $3,209(86)$ \\
\hline Not in line hypothesis & 16 & $147(15)$ & $862(85)$ \\
\hline \multicolumn{4}{|l|}{ Hypothesis HDL-c } \\
\hline In line hypothesis-decrease HDL-c & 86 & $498(13)$ & $3,311(87)$ \\
\hline Not in line hypothesis & 9 & $90(16)$ & $457(84)$ \\
\hline \multicolumn{4}{|l|}{ Hypothesis ratio TC:HDL-C } \\
\hline $\begin{array}{l}\text { In line hypothesis-increase } \\
\text { TC:HDL-C }\end{array}$ & 38 & $211(16)$ & $1,136(84)$ \\
\hline Not in line hypothesis & 2 & $11(14)$ & $65(86)$ \\
\hline \multicolumn{4}{|l|}{ Article characteristics_-content related } \\
\hline \multicolumn{4}{|l|}{ Study design } \\
\hline Observational study & 6 & $18(7)$ & $236(93)$ \\
\hline Experimental study & 36 & $475(23)$ & $1,584(77)$ \\
\hline Systematic review & 9 & $49(21)$ & $183(79)$ \\
\hline Narrative review & 54 & $122(5)$ & $2,329(95)$ \\
\hline Other & 3 & $5(11)$ & $40(89)$ \\
\hline \multicolumn{4}{|l|}{ Sample size ${ }^{c}$} \\
\hline $10-40$ & 22 & $280(20)$ & $1,115(80)$ \\
\hline $40-80$ & 13 & $190(31)$ & $419(69)$ \\
\hline$>80$ & 15 & $91(13)$ & $586(87)$ \\
\hline \multicolumn{4}{|l|}{ Number of extra determinants ${ }^{c}$} \\
\hline 0 & 34 & $120(7)$ & $1,519(93)$ \\
\hline 1 & 21 & $83(13)$ & $534(87)$ \\
\hline$>1$ & 52 & $466(17)$ & $2,319(83)$ \\
\hline \multicolumn{4}{|l|}{ Number of extra outcomes ${ }^{c}$} \\
\hline $0-3$ & 43 & $222(9)$ & $2,186(91)$ \\
\hline $4-6$ & 29 & $267(20)$ & $1,102(80)$ \\
\hline$>6$ & 36 & $180(14)$ & $1,084(86)$ \\
\hline \multicolumn{4}{|l|}{$\begin{array}{l}\text { Article characteristics-Not content } \\
\text { related }\end{array}$} \\
\hline \multicolumn{4}{|l|}{ Number of affiliations ${ }^{c}$} \\
\hline 1 & 36 & $207(11)$ & $1,689(89)$ \\
\hline 2 & 28 & $153(13)$ & $998(87)$ \\
\hline$>2$ & 43 & 309 (15) & $1,685(85)$ \\
\hline \multicolumn{4}{|l|}{ Journal impact factor ${ }^{\mathrm{C}}$} \\
\hline $0-2$ & 31 & $34(2)$ & $1,439(98)$ \\
\hline $2-4$ & 45 & $311(14)$ & $1,886(86)$ \\
\hline$>4$ & 31 & $322(24)$ & $1,020(76)$ \\
\hline
\end{tabular}


Table 1. Continued

\begin{tabular}{|c|c|c|c|}
\hline Article characteristic & N Publications & $N$ citation paths utilized (\%) & $N$ citation paths not utilized (\%) \\
\hline Not-for-profit & 39 & $248(12)$ & $1,730(88)$ \\
\hline For-profit & 13 & $133(19)$ & $570(81)$ \\
\hline Both & 9 & $113(23)$ & $373(77)$ \\
\hline Not reported & 45 & $175(9)$ & $1,699(91)$ \\
\hline \multicolumn{4}{|l|}{ Number of references ${ }^{c}$} \\
\hline$<30$ & 22 & $206(17)$ & $992(83)$ \\
\hline $30-50$ & 43 & $276(14)$ & $1,674(86)$ \\
\hline$>50$ & 42 & $187(10)$ & $1,706(90)$ \\
\hline \multicolumn{4}{|l|}{ Title of publication } \\
\hline Not suggesting a conclusion & 91 & $541(13)$ & $3,734(87)$ \\
\hline Suggesting a conclusion & 17 & $128(17)$ & $638(83)$ \\
\hline \multicolumn{4}{|l|}{ Language } \\
\hline English & 104 & $669(13)$ & $4,291(87)$ \\
\hline Other & 4 & $0(0)$ & $81(100)$ \\
\hline \multicolumn{4}{|l|}{ Author characteristics } \\
\hline \multicolumn{4}{|l|}{ Gender } \\
\hline Male & 74 & $514(15)$ & $2,983(85)$ \\
\hline Female & 33 & $155(10)$ & $1,325(90)$ \\
\hline \multicolumn{4}{|l|}{ Affiliation corresponding author } \\
\hline University & 87 & $508(12)$ & $3,712(88)$ \\
\hline Government & 7 & $87(27)$ & $232(73)$ \\
\hline Industry & 7 & $26(13)$ & $168(87)$ \\
\hline Hospital & 3 & $1(4)$ & $22(96)$ \\
\hline Other & 4 & $4(2)$ & $181(98)$ \\
\hline \multicolumn{4}{|l|}{ Authority ${ }^{c}$} \\
\hline$<10$ & $N A^{b}$ & $128(7)$ & $1,610(93)$ \\
\hline $11-60$ & $N A^{b}$ & $254(17)$ & $1,281(83)$ \\
\hline$>60$ & $N A^{b}$ & $287(16)$ & $1,481(84)$ \\
\hline
\end{tabular}

a Missing values, because of unclear findings or lack of reporting, are not reported in this table.

${ }^{b}$ Values are not measured on publications level but vary over time.

${ }^{c}$ Variables are measured on continuous scale and decoded into categories based on their tertiles.

\subsection{Statistical analysis}

Each publication in the network plays the role of a citing and a cited publication. We were solely interested in the effect of the characteristics of the cited publication on the likelihood of being cited. The unit of analysis in this study was therefore the citation path itself. A potential citation path existed between one publication and every other publication in the network that was published at least 2 years earlier. This limit of 2 years was set to allow the citing publication sufficient time to be written, reviewed, and published. In the constructed data set, each row represented a potential citation path followed by an indication whether the potential citation path had actually been utilized or not and the characteristics of the cited publication of that citation path.

A single publication could cite to multiple other publications. Therefore, a multilevel approach was required, in which the citation paths were nested under the citing publication. Random effect logistic regression was modeled to assess the effect of characteristics of the cited article on the likelihood of being cited. Fixed effect logistic regression analysis was used to test concordance between the cited and citing publication in relation to citation behavior.

First, univariate analyses were performed to test every previously described potential determinant of citation in the cited publication as a predictor of the likelihood of being cited. Second, each analysis was adjusted for study design and sample size. These two variables were used as a proxy for study quality, which we considered to be an acceptable driver of selective citation. All statistical analyses were performed in Stata 13 .

The outcomes of the logistic regression are reported in the results section of this publication as odds ratios. According to the literature, the odds ratio gives an overestimation of the risk compared to the relative risk in studies where the outcome is a common condition [36]. In our network, the prevalence of being cited is 13\% (669 actual citations of 5,041 potential citations). With this prevalence, we do not consider "being cited" as a very common 
condition, and hence the overestimation of the odds ratio over the relative risk will be within considerable limits [36]. Ultimately, the odds ratio gives an accurate estimation of the direction of the effect; only the exact magnitude of the effect should be interpreted with some caution. For the readability of the publication, we interpret these values as if they are relative risks and therefore speak about "the likelihood of being cited."

\section{Results}

In total 108 publications, published between 1990 and 2015, on the effect of IP-TFA on cholesterol were identified in the network. Among these publications, 5,041 potential citation paths existed; 669 of them have been utilized. Characteristics of the network are displayed in Table 1 . Table 2 displays the crude odds ratios for all potential determinants of the likelihood of being cited. Fig. 2 visualizes of part of the network, namely the empirical studies (circles) and systematic reviews (squares). From the visualization, the development of the amount of evidence over time can be seen together with the timing of the systematic reviews. In addition, the visualization shows that some publications are highly cited within the network (e.g., Mensink, 1990), whereas others are less popular (e.g., Mutalib, 1999).

\subsection{Study outcome}

In this network, each narrative review was in line with the hypotheses that IP-TFA increases LDL-c, decreases HDL-c, and increases TC:HDL-c ratio (data not shown).

Reporting a significant effect of trans fat intake on LDLc, regardless of the direction of the effect, is a strong predictor of citation and leads to an approximately three times higher likelihood of being cited. After adjusting for study design and sample size this effect remained, suggesting that the effect cannot be explained by study quality. For the relation between significant findings on HDL-c and the ratio TC:HDL-c and citation, this effect is also present but of a lower magnitude of approximately 1.5 and 2, respectively. Taking into account the direction of the effect, being in line with the hypothesis on LDL-c and HDL-c or not, is a significant determinant of citation only after adjusting for study design and sample size (Table 2). Reporting results that are in line with the hypothesis that TC:HDL-c is increased by IP-TFA intake or not, has no impact on the likelihood of being cited. Because the variables "significance" and "being in line with hypothesis" are related to each other, a Bonferroni adjustment was performed to correct for multiple comparisons. These two variables are tested in six hypotheses, regarding LDL-c, HDL-c, and TC:HDL-c. After applying a Bonferroni correction, an $\alpha$ of 0.008 was taken into account. This did not change the interpretation of the results.

\subsection{Article characteristics-content related}

Large variation was observed in the sample sizes of the publications, ranging from 10 to 140,390 participants. To deal with the large range and nonparametric distribution, sample size was used as a categorical variable in the analysis (Table 1). Naturally, sample size highly correlates with study design, as the lowest category of sample size (0 participants) mainly contains narrative reviews, whereas observational studies only appear in the highest category (more than 80 participants). Nevertheless, there was no proof of collinearity between sample size and study design, and the likelihood of being cited increased with a higher number of participants (Table 2).

Although narrative reviews made up half of the network, they get cited rarely (5\%) compared to every other research design (Table 1). The likelihood of being cited for intervention studies and systematic reviews is 6 and 7 times higher, respectively, compared with narrative reviews (Table 2). However, the effect of experimental study design on citation is explained completely by the sample size, as the odds ratio changed from 6.0 to 0.84 after correcting for sample size (Table 2).

Many publications included additional determinants and outcomes besides IP-TFA and LDL-c and HDL-c (Table 1). The likelihood of being cited increased with both additional determinants and study outcomes, shown by a $P$-trend of $<$ 0.001 for the crude ORs of both variables. This can be explained because less specific publications can be cited for multiple reasons by different research areas. The effect of additional outcomes and determinants on the likelihood of being cited disappeared and even reversed, after adjusting for study design and sample size, respectively.

\subsection{Article characteristics - not content related}

Hedge factors were measured for all publications written in the traditional format (i.e., having separate sections for introduction, method, results, and discussion), which were 42 publications in total. Values of the hedge factor ranged from 0.57 to 3.26 , on a 5 -point scale, with a median value of 1.35 . The likelihood of being cited increased significantly by almost three times with an increasing hedge factor (crude OR: 2.92 95\% CI: 2.1-4.0). As explained before, a higher hedge factor referred to more uncertain statements.

The journal impact factor (JIF) at the moment of publication of an article ranged from 0 to 53 and followed a nonparametric distribution. Therefore, JIF was categorized based on its tertiles (Table 1). Having an impact factor higher than 4 increased the likelihood of being cited by approximately 18 times, compared with an impact factor lower than 2. Because the JIF was measured at the moment of publication, the JIF did not interfere with the dependent variable of citation.

Having a title stating the conclusion significantly raised the likelihood of being cited (OR: 1.46, 95\% CI: 1.2-1.8). 
Table 2. Crude and adjusted odds ratios $(95 \% \mathrm{Cl})$ for the likelihood of being cited

\begin{tabular}{|c|c|c|}
\hline Article characteristic & Crude OR & Adjusted $\mathbf{O R}^{\mathrm{b}}$ \\
\hline \multicolumn{3}{|l|}{ Study outcome } \\
\hline Significance LDL-c ${ }^{a}$ & $3.08(2.4-4.0)$ & $3.15(2.4-4.2)$ \\
\hline Significance HDL-c ${ }^{a}$ & $1.55(1.3-1.9)$ & $1.67(1.3-2.1)$ \\
\hline $\begin{array}{l}\text { Significance ratio TC: HDL- } \\
\mathrm{C}^{\mathrm{a}}\end{array}$ & $1.59(1.1-2.3)$ & $2.21(1.4-3.5)$ \\
\hline Hypothesis LDL-c & $1.06(0.9-1.3)$ & $3.30(2.6-4.2)$ \\
\hline Hypothesis HDL-c & $0.86(0.7-1.1)$ & $2.09(1.6-2.8)$ \\
\hline Hypothesis ratio TC: HDL-C & $1.12(0.6-2.2)$ & $0.35(0.1-2.4)$ \\
\hline \multicolumn{3}{|l|}{$\begin{array}{l}\text { Article } \\
\text { characteristics-content } \\
\text { related }\end{array}$} \\
\hline \multicolumn{3}{|l|}{ Study design ${ }^{c}$} \\
\hline Narrative review & 1 (ref) & 1 (ref) \\
\hline Observational study & $1.15(0.7-2.0)$ & $0.40(0.2-0.8)$ \\
\hline Experimental study & $6.00(4.8-7.5)$ & $0.84(0.3-2.4)$ \\
\hline Systematic review & $7.20(4.9-10.6)$ & $3.41(2.1-5.5)$ \\
\hline$P$ for trend & $<0.001$ & $<0.001$ \\
\hline \multicolumn{3}{|l|}{ Sample size $^{d}$} \\
\hline 0 participants & 1 (ref) & 1 (ref) \\
\hline 1-40 participants & $5.60(4.4-7.1)$ & $7.05(2.5-20.1)$ \\
\hline 41-80 participants & $9.8(7.5-12.9)$ & $12.34(4.3-35.5)$ \\
\hline$>80$ participants & $3.42(2.5-4.6)$ & $3.38(2.2-5.1)$ \\
\hline$P$ for trend & 0.025 & $<0.001$ \\
\hline \multicolumn{3}{|l|}{ Number of determinants } \\
\hline 0 additional determinants & 1 (ref) & 1 (ref) \\
\hline 1 additional determinant & $2.54(1.9-3.5)$ & $0.38(0.2-0.6)$ \\
\hline $\begin{array}{r}>1 \text { additional } \\
\text { determinant }\end{array}$ & $2.70(2.2-3.4)$ & $0.43(0.3-0.6)$ \\
\hline$P$ for trend & $<0.001$ & 0.001 \\
\hline \multicolumn{3}{|l|}{ Number of outcomes } \\
\hline 0-3 additional outcomes & 1 (ref) & 1 (ref) \\
\hline 4-6 additional outcomes & $2.57(2.1-3.2)$ & $1.21(0.9-1.5)$ \\
\hline$>6$ additional outcomes & $2.17(1.7-2.7)$ & $0.92(0.7-1.2)$ \\
\hline$P$ for trend & $<0.001$ & 0.701 \\
\hline \multicolumn{3}{|l|}{$\begin{array}{l}\text { Article characteristics-not } \\
\text { content related }\end{array}$} \\
\hline $\begin{array}{l}\text { Hedge factor Number of } \\
\text { affiliations }\end{array}$ & $2.64(1.9-3.6)$ & $3.27(2.1-5.1)$ \\
\hline 1 affiliation & 1.00 (ref) & 1 (ref) \\
\hline 2 affiliations & $1.36(1.1-1.7)$ & $1.41(1.1-1.8)$ \\
\hline$>2$ affiliations & $1.78(1.5-2.2)$ & $1.12(0.9-1.4)$ \\
\hline$P$ for trend & $<0.001$ & 0.254 \\
\hline \multicolumn{3}{|l|}{ Journal impact factor } \\
\hline $0-2$ & 1 (ref) & 1 (ref) \\
\hline $2-4$ & $7.58(5.2-11.0)$ & $5.51(3.6-8.3)$ \\
\hline$>4$ & $18.0(12.3-26.4)$ & $10.88(7.1-16.6)$ \\
\hline$P$ for trend & $<0.001$ & $<0.001$ \\
\hline \multicolumn{3}{|l|}{ Funding source } \\
\hline For-profit vs not-for-profit & $1.64(1.3-2.1)$ & $1.18(0.9-1.6)$ \\
\hline $\begin{array}{l}\text { Reported vs not reported } \\
\text { funding }\end{array}$ & $0.88(0.7-1.1)$ & $1.10(0.9-1.4)$ \\
\hline
\end{tabular}

Table 2. Continued

\begin{tabular}{|c|c|c|}
\hline Article characteristic & Crude OR & Adjusted $\mathbf{O R}^{\mathrm{b}}$ \\
\hline \multicolumn{3}{|l|}{ Number of references } \\
\hline$<30$ references & 1 (ref) & 1 (ref) \\
\hline $30-50$ references & $0.87(0.7-1.1)$ & $0.74(0.6-0.9)$ \\
\hline$>50$ references & $0.60(0.5-0.8)$ & $0.55(0.4-0.7)$ \\
\hline$P$ for trend & $<0.001$ & $<0.001$ \\
\hline Title of publication & $1.46(1.2-1.8)$ & $1.06(0.8-1.4)$ \\
\hline \multicolumn{3}{|l|}{ Author characteristics } \\
\hline \multicolumn{3}{|l|}{ Gender } \\
\hline Female vs male & $0.72(0.6-0.9)$ & $1.19(0.9-1.6)$ \\
\hline \multicolumn{3}{|l|}{$\begin{array}{l}\text { Affiliation corresponding } \\
\text { author }\end{array}$} \\
\hline Public vs private sector & $1.37(0.9-2.1)$ & $0.83(0.5-1.3)$ \\
\hline \multicolumn{3}{|l|}{ Authority } \\
\hline$<10$ & 1 (ref) & 1 (ref) \\
\hline $11-60$ & $3.15(2.4-4.1)$ & $2.70(1.9-3.8)$ \\
\hline$>60$ & $4.76(3.6-6.2)$ & $5.06(3.5-7.4)$ \\
\hline$P$ for trend & $<0.001$ & $<0.001$ \\
\hline
\end{tabular}

a Odds ratio of chance to be cited for significant vs not significant findings.

b Model adjusted for study design and sample size, both as categorical variables.

${ }^{c}$ Adjusted model is adjusted for sample size.

${ }^{d}$ Adjusted model is adjusted for study design.

This effect reduced to a nonsignificant OR of 1.06 after adjusting for sample size and study design. Regarding language, only four publications were identified that were written in a language other than English and none of them received any citations in this network (Table 1). Therefore, language could not be included in the analysis as a potential determinant of citation. Looking at funding of the study, which the publication reports, a large part of the network (45 publications) did not report any funding source. Nevertheless, no significant effect of reporting funding on the likelihood of being cited was found (Table 2). When comparing different types of funding, for-profit funding had a 1.64 times higher likelihood of being cited compared with not-for-profit funding in this network (Table 2).

The number of references in a publication is inversely related to the likelihood of being cited. This contradicts our hypothesis that a higher number of references would lead to more credibility and therefore more citations. The number of references is often limited by the journal, and this rule might be different for high impact factor journals than low impact factor journals. However, the Pearson's correlation between the JIF and number of citations is only 0.0296 .

\subsection{Author characteristics}

The majority of publications in this network had a male corresponding author (Table 1). The corresponding authors 


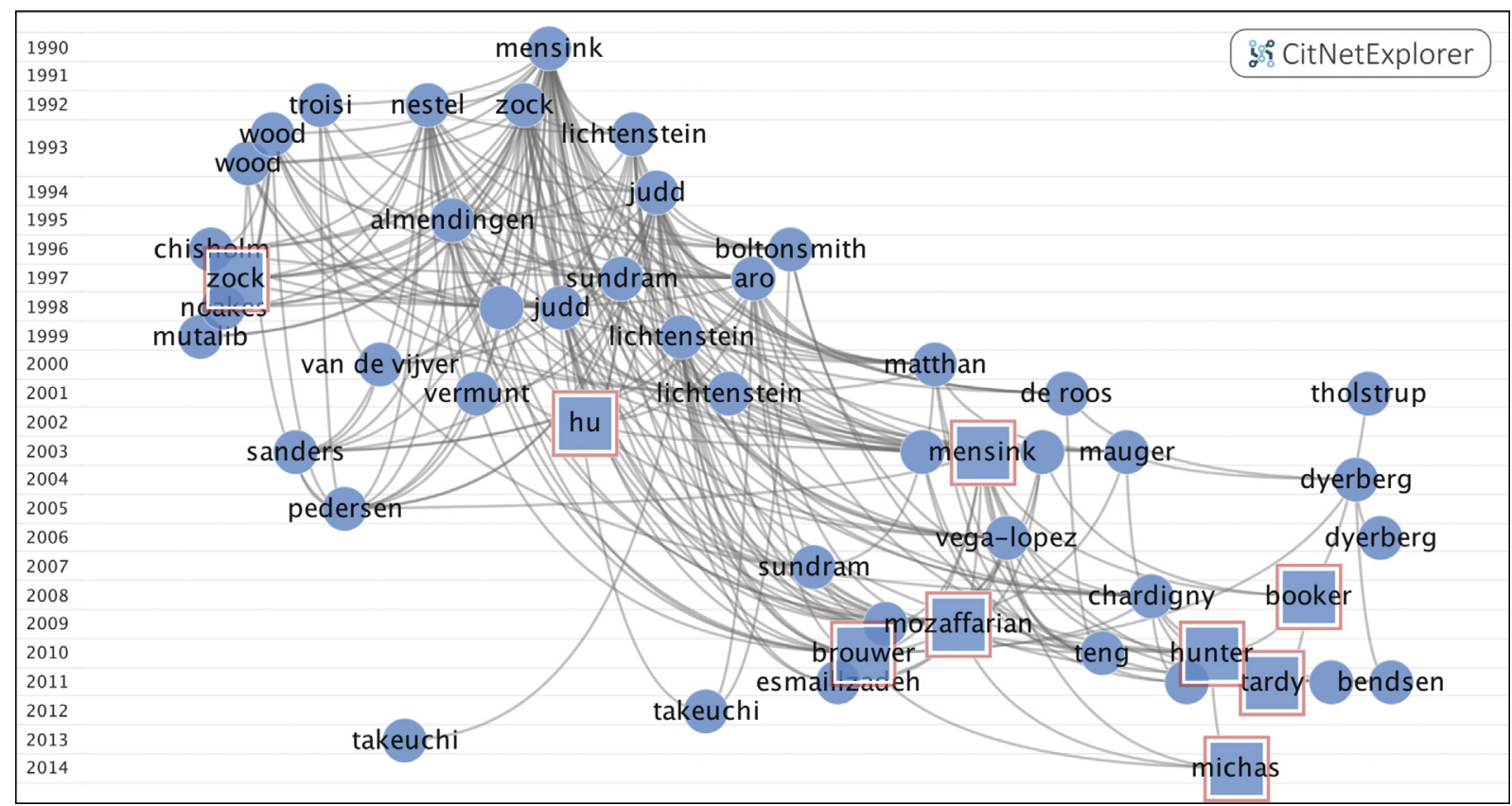

Fig. 2. Visualization of the empirical studies and systematic reviews in the network* * Circles reflect the empirical studies; squares reflect systematic reviews. Each line depicts a performed citation. The $y$-axis shows the time line; the $x$-axis is only for visualization purposes.

of 87 publications were affiliated to a university. The likelihood of being cited is significantly smaller for female corresponding authors compared with male corresponding authors (OR: 0.72; 95\% CI: 0.6-0.9), whereas the affiliation of the corresponding author did not make a difference (OR: 1.37; 95\% CI: 0.9-2.1). After correcting for study design and sample size, both gender (OR: 1.19; 95\% CI: 0.9-1.6) and affiliation (OR: 0.83; 95\% CI: 0.5-1.3) did not significantly influence the likelihood of being cited anymore. The authority of the authors, which is determined by the total number of citations received each year in the network, ranged from 0 to 255 and followed a skewed distribution (median: 30; IQR: 3-93). Based on the tertiles, the variable was recoded into a categorical variable with three categories $(<10,11-60,>60)$. As expected, the likelihood of being cited increased three- to four-fold with an increase in the authority of the authors. Also after adjusting for study design and sample size, this significant effect of authority on the likelihood of being cited remained and even increased to an OR of 5 (Table 2).

To assess the impact of the large number of narrative reviews in this network, a sensitivity analysis was performed leaving out the narrative reviews as cited publications. This did not result in a substantial shift in outcomes, compared to the overall crude and adjusted analysis. Results of this analysis are presented in Table $\mathrm{S} 1$ in the Digital Supplement.

Results of the concordance analyses are presented in Table S2 of the Digital Supplement. It appeared that concordance between the citing and cited publication did not greatly influence the likelihood of citation for any of the study characteristics.

\section{Discussion}

In this network, we found that several factors are determinants for selective citation. First, citation bias was found because the adjusted analysis showed a significantly higher likelihood of citation for statistically significant studies on the relationship between LDL-c, HDL-c and TC:HDL-c, and serum cholesterol. Citation bias can affect the scientific process in several ways. For example, it might drive the reader, consciously or unconsciously, in a certain direction. Especially when this happens systematically, the reader might develop a certain belief system that is not in line with the available evidence. The effect of citation bias is especially dangerous because it cannot easily be detected within one publication. By systematic overrepresentation of and exposure to positive results, gradually a shift toward an unfounded belief system might be made. Greenberg (2009) showed in his citation analysis that citation bias can amplify certain claims by citing to reviews, which lack data addressing this claim [23]. As a kind of mere exposure effect, the reader will accept this claim more easily. The harmful effect of citation bias was shown clearly by Ioannidis (2011). This study showed that highly cited publications show exaggerated effect sizes when compared with effect sizes of meta-analyses in the same field [24]. Consequently, these exaggerated effect sizes will be of great influence in the research field because of their high number of citations, whereas they do not reflect the true effect.

Citation bias has been studied together with other types of selective reporting, such as outcome reporting bias and publication bias [37]. It is seen as a general phenomenon that negative findings receive less attention, which leads 
to less publication, reporting and citation of negative or nonsignificant results [37]. All types of selective reporting lead to an overrepresentation of certain evidence, making it very difficult to indicate the true effect and even potentially threatening the validity of meta-analyses [38,39]. Although meta-analyses will be primarily influenced by publication bias and outcome reporting bias, also citation bias might play a role in this. Identification of literature via reference checking and contacting experts in the field are fixed components of systematic reviews and metaanalyses. Negative studies that are not often cited might be missed in this process, compared to positive, highly cited publications. Particularly in narrative reviews, citation bias might affect the discussion. Scientific knowledge development and agenda setting might be seriously influenced by over-reporting certain results, while neglecting other evidence. This becomes particularly problematic when scientific consensus is being used as a basis for clinical guidelines, legislation, industry decisions, or future research funding.

Additional to citation bias, our study identified other determinants of selective citation. Especially sample size, JIF and the authority of the author were positively correlated with the likelihood of being cited. These findings were in line with previous literature. Onodera (2013) showed that the influence of JIF is greater than author-related factors, such as the number of citations received by each author, with regard to citation rates [26]. Callaham (2002) analyzed which factors influenced the citation of publications for which abstracts had been submitted to a scientific meeting [40]. In this body of literature, sample size was the biggest predictor of citation, whereas study outcome had no effect on citation. Opposed to previous research, factors such as funding and the title of the publication were not associated with citation in the current network.

The methodology used in this work is different from earlier citation analyses in several ways. Previous studies have often compared the citation count toward positive and negative study (e.g., 41). We have however assessed the likelihood of being cited for publications, in relation to a number of determinants, by taking into account all potential citation pathways. In addition, the statistical method, a multilevel logistic regression, has not been used on previous citation analyses. With this approach we take into account the fact that citations are clustered in publications, and therefore, not all citation pathways are independent of each other. Finally, we studied not only the characteristics of the cited publication in relation to the chance of citation but we also looked at concordance. The rational behind this analysis was that authors use citations to amplify their own findings, and therefore cite mostly to sources that share the same characteristics. This would be different form of selective citation.

Our study also has several limitations. First, the search strategy to map the network was only conducted on the Web of Science database, making it impossible to use
MeSH-terms in the search strategy and potentially leaving relevant publications unidentified. Especially the fact that only four non-English publications were identified, makes us suspect that not all relevant publications have been found. The reason for limiting the search to the WoS database was that this is the only database that enabled us to download the publications together with all corresponding citation paths, which was required for developing the data set. Second, the study outcome of each publication, which could be either statistical significance or being in line with the hypothesis, was scored based on the evidence reported in the result section of the publication. This does not necessary correspond with the way the publication has been cited by other publications. Although it is likely that citations are based on the presented evidence, it might also be based on the author's overall conclusion. The third limitation relates to the statistical analysis. Initially, it was planned to perform a multivariate analysis, to adjust for all determinants that might relate to citation frequency. In this way, we aimed to develop a model to predict the chance of being cited. In the prespecified study protocol, it was laid down that the multivariate model would include all determinants that showed a significant effect on the likelihood of being cited in the crude analysis. Unfortunately, analyzing this multivariate model created a very unstable model. As it was impossible to draw meaningful conclusions from this analysis, we decided to limit our study to the analysis adjusted for study design and samples size, as a proxy for study quality. Also in the adjusted analysis we should be aware that there might be overlap in the explained variation by both study design and sample size, as these variables are closely related. Nevertheless, no evidence for collinearity between these two variables was found.

In conclusion, with this study we have shown a new methodology to assess the occurrence of citation bias, as well as created a broader insight into the influence of other determinants of selective citation. From previous research, citation bias has shown to be a problem in several research fields. Also in the example study on trans fatty acid intake and serum cholesterol, citation bias was found. Sample size, JIF, and authority of the author were identified as other important determinants of citation. Further studies are needed to confirm these findings in other research areas and assess the consequences of selective citation for knowledge development and science-based decision-making.

\section{Acknowledgments}

The authors thank prof Ronald Mensink for his advice on defining the research area. The authors also thank Halil Kilicoglu for calculating the hedge factors for this study.

This project has received funding from the Eatwell project of Maastricht University. Maastricht University had no role in study design, data collection and analysis, preparation of the article, or decision to publish. 
Authors' contributions: M.J.E.U., B.D., G.M.H.S., L.M.B., and M.P.A.Z. have been involved in the development of the study design. Data collection was performed by M.J.E.U., B.D., and G.M.H.S. Data analysis was performed by M.J.E.U. All coauthors were actively involved in the interpretation of the data. Drafting the article was performed by M.J.E.U.; the article was critically revised by all coauthors. All coauthors approved the final version of the article to be published.

\section{Supplementary data}

Supplementary data related to this article can be found at https://doi.org/10.1016/j.jclinepi.2018.10.008.

\section{References}

[1] Merton RK. The Matthew effect in science. Science 1968;159: $56-63$.

[2] Bornmann L, Daniel H. What do citations measure? A review of studies on citing behavior. J Doc 2006;64(1):45-80.

[3] Zuckerman H. Citation analysis and the complex problem of intellectual influence. Scientometrics 1987;12(5-6):329-38.

[4] Gilbert GN. Referencing as persuasion. Soc Stud Sci 1977;7:113-22.

[5] Cole J, Cole S. Measuring the quality of sociological research: problems in the use of the science citation index. Am Sociol 1971;6:23-9.

[6] Moed HF. Citation analysis in research evaluation. Dordrecht, The Netherlands: Springer Science \& Business Media; 2006.

[7] Robinson KA, Dunn AG, Tsafnat G, Glasziou P. Citation networks of related trials are often disconnected: implications for bidirectional citation searches. J Clin Epidemiol 2014;67:793-9.

[8] Robinson KA, Goodman SN. A systematic examination of the citation of prior research in reports of randomized, controlled trials. Ann Intern Med 2011;154:50-5.

[9] Song F, Parekh S, Hooper L, Loke YK, Ryder J. Dissemination and publication of research findings: an updated review of related biases. Health Technol Assess 2010;14:234.

[10] Fanelli D. How many scientists fabricate and falsify research? A systematic review and meta-analysis of survey data. PLoS One 2009;4:e5738.

[11] Bouter LM, Tijdink J, Axelsen N, Martinson BC, ter Riet G. Ranking major and minor research misbehaviors: results from a survey among participants of four World Conferences on Research Integrity. Res Integr Peer Rev 2016;1(1):17.

[12] Duyx B, Urlings MJ, Swaen GM, Bouter LM, Zeegers MP. Scientific citations favor positive results: a systematic review and meta-analysis. J Clin Epidemiol 2017;88:92-101.

[13] Ravnskov U. Cholesterol lowering trials in coronary heart disease: frequency of citation and outcome. BMJ 1992;305(6844):15-9.

[14] Trinquart L, Johns DM, Galea S. Why do we think we know what we know? A metaknowledge analysis of the salt controversy. Int J Epidemiol 2016;45:251-60.

[15] Schumm WR. Evidence of pro-homosexual bias in social science: citation rates and research on lesbian parenting. Psychol Rep 2010; 106(2):374-80.

[16] Andrade NS, Flynn JP, Bartanusz V. Twenty-year perspective of randomized controlled trials for surgery of chronic nonspecific low back pain: citation bias and tangential knowledge. Spine J 2013;13(11):1698-704.
[17] Kjaergard LL, Gluud C. Citation bias of hepato-biliary randomized clinical trials. J Clin Epidemiol 2002;55:407-10.

[18] Nieminen P, Rucker G, Miettunen J, Carpenter J, Schumacher M. Statistically significant papers in psychiatry were cited more often than others. J Clin Epidemiol 2007;60:939-46.

[19] Kostoff R. The difference between highly and poorly cited medical articles in the journal Lancet. Scientometrics 2007;72(3):513-20.

[20] Jannot A-S, Agoritsas T, Gayet-Ageron A, Perneger TV. Citation bias favoring statistically significant studies was present in medical research. J Clin Epidemiol 2013;66:296-301.

[21] Leimu R, Koricheva J. What determines the citation frequency of ecological papers? Trends Ecol Evol 2005;20(1):28-32.

[22] Etter J-F, Stapleton J. Citations to trials of nicotine replacement therapy were biased toward positive results and high-impact-factor journals. J Clin Epidemiol 2009;62:831-7.

[23] Greenberg SA. How citation distortions create unfounded authority: analysis of a citation network. BMJ 2009;339:b2680.

[24] Ioannidis JPA, Panagiotou OA. Comparison of effect sizes associated with biomarkers reported in highly cited individual articles and in subsequent meta-analyses. JAMA 2011;305:2200-10.

[25] Bornmann L, Daniel H-D. What do citation counts measure? A review of studies on citing behavior. J Doc 2008;64(1):45-80.

[26] Onodera N, Yoshikane F. Factors affecting citation rates of research articles. J Assoc Inf Sci Technol 2015;66(4):739-64.

[27] Sismondo S. How pharmaceutical industry funding affects trial outcomes: causal structures and responses. Soc Sci Med 2008;66(9): 1909-14

[28] Conen D, Torres J, Ridker PM. Differential citation rates of major cardiovascular clinical trials according to source of funding: a survey from 2000 to 2005. Circulation 2008;118:1321-7.

[29] Kilicoglu H, Bergler S. Recognizing speculative language in biomedical research articles: a linguistically motivated perspective. BMC Bioinformatics 2008;9(11):1.

[30] Mensink RP, Katan MB. Effect of dietary trans fatty acids on highdensity and low-density lipoprotein cholesterol levels in healthy subjects. N Engl J Med 1990;323(7):439-45.

[31] Booker C, Mann J. Trans fatty acids and cardiovascular health: translation of the evidence base. Nutr Metab Cardiovasc Dis 2008;18(6): $448-56$.

[32] Hu FB, Willett WC. Optimal diets for prevention of coronary heart disease. JAMA 2002;288:2569-78.

[33] Hunter JE, Zhang J, Kris-Etherton PM. Cardiovascular disease risk of dietary stearic acid compared with trans, other saturated, and unsaturated fatty acids: a systematic review. Am J Clin Nutr 2010;91(1): 46-63.

[34] Okie S. New York to trans fats: you're out!. N Engl J Med 2007; 356(20):2017-21.

[35] Astrup A. The trans fatty acid story in Denmark. Atheroscler Suppl 2006;7(2):43-6.

[36] Cummings P. The relative merits of risk ratios and odds ratios. Arch Pediatr Adolesc Med 2009;163(5):438-45.

[37] Fanelli D. Positive results receive more citations, but only in some disciplines. Scientometrics 2013;94(2):701-9.

[38] Thornton A, Lee P. Publication bias in meta-analysis: its causes and consequences. J Clin Epidemiol 2000;53:207-16.

[39] Dwan K, Altman DG, Arnaiz JA, Bloom J, Chan A-W, Cronin E, et al. Systematic review of the empirical evidence of study publication bias and outcome reporting bias. PLoS One 2008;3:e3081.

[40] Callaham M, Wears RL, Weber E. Journal prestige, publication bias, and other characteristics associated with citation of published studies in peer-reviewed journals. JAMA 2002;287:2847-50. 